\title{
Relationship between the transcriptional expression of PIM1 and local control in patients with head and neck squamous cell carcinomas treated with radiotherapy
}

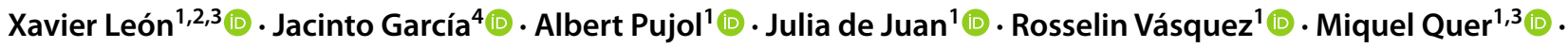 \\ Mercedes Camacho 5 (D)
}

Received: 5 September 2021 / Accepted: 13 December 2021 / Published online: 6 January 2022

(c) Crown 2022

\begin{abstract}
Purpose Proviral integration site for Moloney murine leukemia virus (PIMs) are proto-oncogenes encoding serine/threonine kinases that phosphorylate a variety of substrates involved in the regulation of cellular processes. Elevated expression of PIM-1 has been associated with poor prognosis in several types of cancer. There are no studies that have analyzed the response to radiotherapy in patients with head and neck squamous cell carcinoma (HNSCC) according to the expression of PIM-1. The aim of our study was to analyze the relationship between the transcriptional expression of PIM-1 and local response to radiotherapy in HNSCC patients.

Methods We determined the transcriptional expression of PIM-1 in 135 HNSCC patients treated with radiotherapy, including patients treated with chemoradiotherapy $(n=65)$ and bioradiotherapy $(n=15)$.

Results During the follow-up, 48 patients (35.6\%) had a local recurrence of the tumor. Patients with local recurrence had a higher level of PIM-1 expression than those who achieved local control of the disease $(P=0.017)$. Five-year local recurrencefree survival for patients with a high expression of PIM-1 $(n=43)$ was $44.6 \%$ (95\% CI 29.2-60.0\%), and for patients with low expression $(n=92)$ it was $71.9 \%(95 \%$ CI 62.5-81.3\%) $(P=0.007)$. According to the results of multivariate analysis, patients with a high PIM-1 expression had a 2.2-fold increased risk of local recurrence (95\% CI 1.22-4.10, $P=0.009)$.

Conclusion Patients with elevated transcriptional expression levels of PIM-1 had a significantly higher risk of local recurrence after radiotherapy.
\end{abstract}

Keyword PIM-1 $\cdot$ Head and neck carcinoma $\cdot$ Radiotherapy $\cdot$ Local control $\cdot$ Biomarker

Xavier León

xleon@santpau.cat

1 Otorhinolaryngology Department, Hospital de La Santa Creu I Sant Pau, Universitat Autònoma de Barcelona, C/Mas Casanovas, 90, 08041 Barcelona, Spain

2 UVIC. Universitat Central de Catalunya, Barcelona, Vic, Spain

3 Centro de Investigación Biomédica en Red de Bioingeniería, Biomateriales Y Nanomedicina (CIBER-BBN), Madrid, Spain

4 Otorhinolaryngology Department, Hospital del Mar, Universitat Autònoma de Barcelona, Barcelona, Spain

5 Genomics of Complex Diseases, Research Institute Hospital Sant Pau, IIB Sant Pau, Barcelona, Spain

\section{Introduction}

Radiotherapy is one of the therapeutic options for patients with head and neck squamous cell carcinoma (HNSCC). After treatment with radiotherapy, a variable percentage of patients, depending on the location and extension of the tumor, have a local recurrence. The availability of biomarkers with the capacity to discriminate tumor radiosensitivity would make it possible to offer radiotherapy to those patients with tumors with a greater probability of response, reducing the percentage of local recurrence and the need for salvage surgery.

Proviral integration site for Moloney murine leukemia virus (PIMs) are proto-oncogenes encoding serine/threonine kinases that phosphorylate a variety of substrates involved in the regulation of cellular processes such as proliferation, survival, apoptosis, migration, and cell cycle regulation. The 
PIM family consists of three members, PIM-1, PIM-2, and PIM-3 [1].

Elevated expression of PIM-1 has been associated with poor prognosis in patients with leukemia [2] or lymphoma [3], as well as in patients with squamous cell carcinomas located in the esophagus [4] or the lung [5], or adenocarcinomas located in the breast [6], lung [5], pancreas [7], colon [8], stomach [9] or prostate [10].

Studies in experimental models of lung carcinoma [11] or pancreatic carcinoma [12] have shown that PIM-1 expression is associated with a reduction in radiosensitivity. In a study performed with HNSCC cell lines, Peltola et al. [13] demonstrated that PIM-1 expression protects tumor cells from radiotherapy-induced damage. To our knowledge, there are no studies that have analyzed the response to radiotherapy in patients with HNSCC according to the expression of PIM-1.

The aim of our study was to evaluate the relationship between PIM-1 transcriptional expression and sensitivity to radiotherapy in a cohort of patients with HNSCC.

\section{Materials and methods}

\section{Patients}

We carried out a retrospective study based on the analysis of biopsies obtained from the primary location of the tumor prior to any type of treatment in a cohort of 135 patients with a histologically confirmed squamous cell carcinoma located in the oropharynx, hypopharynx or larynx, and treated with radiotherapy during the period 2008-2016. Patients who received concomitant treatment with chemotherapy (chemoradiotherapy) or cetuximab (bioradiotherapy) were also included in the study. Clinical data were obtained from a database that prospectively collects information on all patients with a malignant head and neck tumor treated at our center since 1985 [14].

All patients included in the study were evaluated by the Oncologic Committee of our institution, which proposed the treatment according to the center's clinical guidelines. Table 1 shows the characteristics of the patients included in the study. Given the interaction in tobacco and alcohol consumption, a combined variable of toxics consumption was created with three categories: no consumption; moderate consumption ( $<20$ cigarettes/day and/or $<80$ g alcohol/day); and severe consumption ( $\geq 20$ cigarettes/day and/ or $\geq 80 \mathrm{~g}$ alcohol/day). The HPV status of patients with oropharyngeal carcinoma was determined by viral DNA detection with SPF-10 RT-PCR, using the LiPA25_vl reverse hybridization assay for genotyping. The loco-regional extension category of patients with HPV-positive oropharyngeal
Table 1 Characteristics of the patients included in the study

\begin{tabular}{|c|c|}
\hline & $N(\%)$ \\
\hline Mean age (standard deviation) years & $63.6(11.7)$ \\
\hline \multicolumn{2}{|l|}{ Gender } \\
\hline Men & $120(88.9)$ \\
\hline Women & $15(11.1)$ \\
\hline \multicolumn{2}{|l|}{ Age } \\
\hline$<65$ years & $75(55.6)$ \\
\hline$\geq 65$ years & $60(44.4)$ \\
\hline \multicolumn{2}{|l|}{ Toxic consumption } \\
\hline None & $14(10.4)$ \\
\hline Moderate & $20(14.8)$ \\
\hline Severe & $101(74.8)$ \\
\hline \multicolumn{2}{|l|}{ Location } \\
\hline Oropharynx & $63(46.7)$ \\
\hline Hypopharynx & $16(11.8)$ \\
\hline Larynx & $56(41.5)$ \\
\hline \multicolumn{2}{|l|}{ Local extension } \\
\hline cT1-T2 & $74(54.8)$ \\
\hline cT3-T4 & $61(45.2)$ \\
\hline \multicolumn{2}{|l|}{ Regional extension } \\
\hline cNO & $81(60.0)$ \\
\hline $\mathrm{cN} 1$ & $22(16.3)$ \\
\hline $\mathrm{cN} 2$ & $30(22.2)$ \\
\hline cN3 & $2(1.5)$ \\
\hline \multicolumn{2}{|l|}{ Histologic grade } \\
\hline Well differentiated & $13(9.6)$ \\
\hline Moderately differentiated & $109(80.7)$ \\
\hline Poorly differentiated & $13(9.6)$ \\
\hline \multicolumn{2}{|l|}{ Treatment } \\
\hline Radiotherapy & $55(40.7)$ \\
\hline Chemoradiotherapy & $65(48.2)$ \\
\hline Bioradiotherapy & $15(11.1)$ \\
\hline
\end{tabular}

tumors was reclassified according to the 8th edition of the TNM.

Treatment with radiotherapy consisted of the administration of a dose of 70-72 Gy on the primary location of the tumor, $50 \mathrm{~Gy}$ on the lymph node areas at risk in cN0 patients, and 70-72 Gy on the affected lymph node areas in $\mathrm{cN}+$ patients. Radiotherapy planning was done using 3D conformal fields until 2010, and IMRT (Instensity_Modulated Radiation Therapy) from 2011 onwards, with a linear accelerator as the radiation source. Most patients were treated with standard fractionation $(2 \mathrm{~Gy} /$ fraction, 1 fraction/day, 5 days/week). Thirteen patients (9.6\%) followed a hyperfractionation regimen $(1.2 \mathrm{~Gy} /$ fraction, 2 fractions/ day, 5 days/week). Sixty-five patients had a concomitant treatment with chemoradiotherapy and 15 bioradiotherapy with cetuximab. Patients treated with chemoradiotherapy received between two and three cycles of cisplatin at a dose 
of $100 \mathrm{mg} / \mathrm{m}^{2}$ every three weeks $(n=55)$, or carboplatin 1.5 AUC every week $(n=10)$. Those treated with bioradiotherapy received cetuximab $400 \mathrm{mg} / \mathrm{m}^{2}$ on day 1 of the week preceding radiotherapy, and a weekly dose of $250 \mathrm{mg} / \mathrm{m}^{2}$ cetuximab during radiotherapy. Seventeen patients were treated with uni or bilateral neck dissections after completing radiotherapy.

All patients included in the study had a follow-up of more than 2 years. The mean follow-up period was 5.4 years (standard deviation 3.3 years).

The study was approved by the Institutional Review Committee and was conducted following the principles established in the Declaration of Helsinki.

\section{Transcriptional analysis}

The biopsy samples obtained from each patient were immediately included in RNA-later (Quiagen $\mathrm{GmbH}$, Hilden, Germany) to prevent RNA degradation and stored at $-80{ }^{\circ} \mathrm{C}$ until processing. Total RNA was extracted using Trizol (Invitrogen, Carlsbad, USA) according to the manufacturer's instructions. The cDNA was obtained by reverse transcription of $1 \mu \mathrm{g}$ of RNA with the High-Capacity cDNA Archive Kit (Applied Biosystems, Foster City, USA), and the transcriptional expression of PIM-1 and beta-actin as an endogenous control was evaluated by RT-PCR in an ABI Prism 7000 using validated pre-designed assays (TaqMan Gene Expression Assays; Applied Biosystems).

\section{Statistical analysis}

We compared the expression levels of PIM-1 according to gender, age, toxics consumption, location of the primary tumor, local and regional extension of the tumor, histological grade, and the local control of the tumor after treatment with radiotherapy. The distribution of the PIM-1 expression values did not meet the normality criteria, hence the non-parametric techniques of Mann-Whitney and Kruskal-Wallis were used in the comparison of the PIM-1 expression levels. For patients with oropharyngeal tumors, PIM-1 expression was analyzed in relation to the HPV status. The continuous value of PIM-1 expression was categorized according to the local disease control after treatment with radiotherapy with a recursive partitioning analysis, using the CRT (Classification and Regression-Tree) model. We calculated the local recurrence-free survival according to the categories defined by the recursive partitioning analysis with the Kaplan-Meier method, using the log-rank test in the comparison of the survival curves. A multivariate analysis was carried out with the Cox proportional hazards model, considering the local recurrence-free survival as the dependent variable, and the location of the primary tumor, the local (cT1-2 versus cT34) and regional $(\mathrm{cNO}$ versus $\mathrm{cN}+$ ) extension of the tumor, the histological grade, as well as the expression category of PIM-1 as independent variables.

\section{Results}

There were no significant differences in the transcriptional expression of PIM-1 according to sex $(P=0.941)$, age $(P=0.060)$, toxics consumption $(P=0.301)$, location of the primary tumor $(P=0.092)$, local $(P=0.326)$ or regional $(P=0.896)$ extension of the tumor, or histological grade $(P=0.781)$. Information regarding HPV status was available for 47 of the patients with oropharyngeal carcinomas. The percentage of patients with HPV-positive oropharyngeal carcinomas was $25.5 \%$. No significant differences appeared in PIM-1 expression values as a function of HPV status $(P=0.137)$. Table 1 of the supplementary material shows the median value of the transcriptional expression of PIM-1 according to the variables analyzed.

During the follow-up period, 48 patients $(35.6 \%)$ had a local recurrence of the tumor, 16 patients $(11.9 \%)$ had a regional recurrence, and 15 patients (11.1\%) had distant metastases. Patients with a local tumor recurrence had significantly higher PIM-1 transcriptional expression values than patients in which treatment with radiotherapy achieved local control of the disease $(P=0.017)$. Figure 1 in the supplementary material shows the distribution of the PIM-1 transcriptional expression values according to the local control of the tumor after treatment with radiotherapy.

The recursive partitioning analysis classified the patients into two groups according to the local control of the tumor after treatment with radiotherapy. Patients with a high PIM-1 expression value $(n=43,31.9 \%)$ had a significantly higher rate of local tumor recurrence than

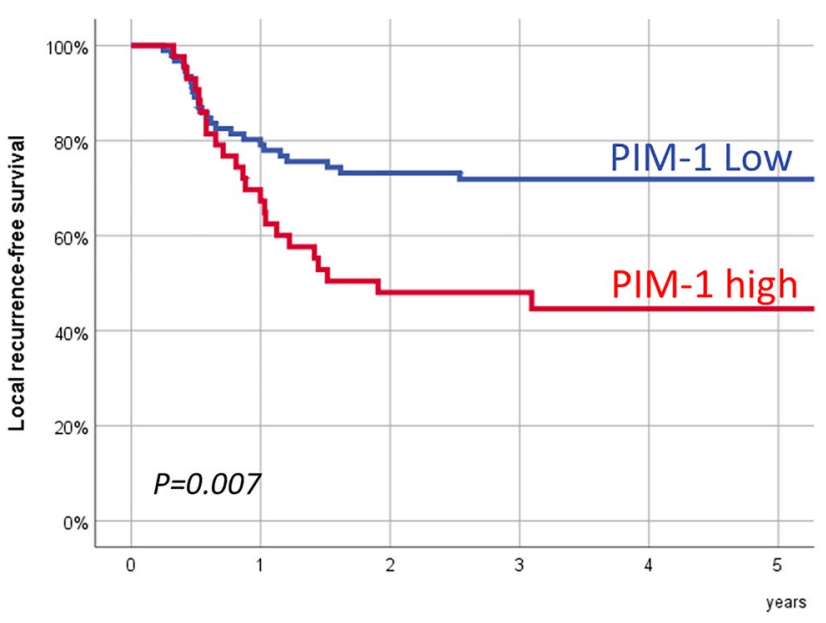

Fig. 1 Local recurrence-free survival according to the PIM-1 expression category 
patients with low expression $(n=92,68.1 \%)$. Five-year local recurrence-free survival for patients with a high PIM-1 expression was $44.6 \%$ (95\% CI 29.2-60.0\%), and for patients with low expression it was $71.9 \%$ (95\% CI 62.5-81.3\%). Figure 1 shows local recurrence-free survival according to the PIM-1 expression categories. There were significant differences in the local recurrence-free survival after treatment with radiotherapy as a function of the PIM-1 expression category $(P=0.007)$.

There were no significant differences in specific-disease survival according to the PIM-1 expression category. Five-year specific-disease survival for patients with a high PIM-1 expression category was $61.1 \%$ (95\% CI 44.3-77.9\%), and for patients with a low expression it was $71.5 \%$ (95 CI 62.1-80.9\%) $(P=0.810)$. There were also no significant differences in regional $(P=0.50)$ or distant disease $(P=0.275)$ recurrence-free survival as a function of the PIM-1 expression.

Table 2 shows the results of multivariate analysis in which local recurrence-free survival after radiotherapy was considered as the dependent variable. The variables that were associated with an increased risk of local recurrence were the location of the tumor in the hypopharynx, an advanced local extension (cT3-T4), and an elevated PMI-1 transcriptional expression category. Considering those patients with a low expression of PIM-1 as the reference category, patients with high expression had a 2.2fold increased risk of local recurrence (95\% CI 1.22-4.10, $P=0.009$ ).

Table 2 Results of a multivariate analysis considering the local control after treatment with radiotherapy as the dependent variable

\begin{tabular}{llll}
\hline & HR & CI 95\% HR & $P$ \\
\hline $\begin{array}{l}\text { Location } \\
\text { Oropharynx }\end{array}$ & 1 & & \\
$\quad$ Hypopharynx & 2.98 & $1.14-7.80$ & 0.026 \\
$\quad$ Larynx & 1.58 & $0.64-3.91$ & 0.315 \\
Local extension & & & \\
cT1-2 & 1 & & \\
cT3-4 & 2.92 & $1.39-6.10$ & 0.004 \\
Regional extension & & & \\
cN0 & 1 & & 0.110 \\
cN+ & 1.98 & $0.85-4.61$ & \\
Treatment & & & 0.930 \\
Radiotherapy & 1 & & 0.479 \\
Chemoradiotherapy & 0.95 & $0.33-2.74$ & \\
Bioradiotherapy & 1.44 & $0.52-4.00$ & \\
PIM-1 & & & 0.009 \\
Low & 1 & & \\
High & 2.24 & $1.22-4.10$ & \\
\hline
\end{tabular}

\section{Discussion}

According to our results, the transcriptional expression of PIM-1 was significantly related to the local control of the tumor in patients with HNSCC treated with radiotherapy, chemoradiotherapy, or bioradiotherapy. Those patients with elevated expression levels of PIM-1 had a 2.24-fold increased risk of local recurrence.

Immunohistochemical studies carried out in patients with HNSCC have found a significant increase in the expression of PIM-1 in tumor tissue, opposite to healthy mucosa $[13,15,16]$. In agreement with the results obtained by other authors that have analyzed the immunohistochemical expression of PIM-1 in HNSCC tumors $[13,15,16]$, we did not find a relationship between the transcriptional expression of PIM-1 and clinicopathological variables such as tumor extension or histological grade.

Regarding the prognostic capacity of PIM-1 in patients with HNSCC, the studies performed so far have mostly included patients treated surgically. In a study of 36 patients with oral cavity carcinomas treated with surgery, Chiang et al. [15] found that patients with elevated PIM-1 expression at transcriptional or immunohistochemical level had worse survival, but the differences did not reach statistical significance. Similarly, in a study by Peltola et al. [13] involving 71 patients with HNSCC treated with surgery and/or radiotherapy, those patients with elevated immunohistochemical expression of PIM-1 tended to have poorer survival $(P=0.09)$. Finally, in 39 patients with carcinoma of the tongue treated with surgery, Tanaka et al. [17] found that immunopositivity to PIM-1 correlated with regional and distant recurrences along with a decrease in survival.

In vitro studies performed with HNSCC cell lines have associated PIM-1 with increased tumor aggressiveness. PIM-1 expression has been associated with increased cell proliferation $[18,19]$, increased migration and invasion capacity $[17,20]$, and increased cell viability and resistance to apoptosis $[18,19]$. In an in vivo experimental study with a murine model, it was shown that inhibition of PIM-1 led to a significant reduction in tumor growth [18]. In nasopharyngeal carcinoma cell lines, PIM-1 inhibition decreased cell proliferation and migration capacity [21].

In tumor models of lung carcinoma [11] or pancreatic carcinoma [12], PIM-1 expression has been associated with a decrease in radiosensitivity. Peltola et al. [13] observed that in patients with HNSCC treated with preoperative radiotherapy, a high expression of PIM-1 correlated with a reduced response to radiotherapy. In HNSCC cell lines, these authors demonstrated that inhibition of PIM-1 expression with siRNA induced radiosensitization of tumor cells, therefore supporting the concept that PIM-1 
protects cells from radiotherapy-induced damage. Similarly, in prostate carcinoma cell lines it has been shown that the increased sensitivity to radiotherapy mediated by miR-124 and miR-144 was a consequence of PIM-1 downregulation [22].

In addition, an association has been described between PMI-1 expression and resistance to chemotherapy in prostate carcinoma models [23]. There is also evidence that downregulation of PIM-1 activity increased sensitivity to chemotherapy in the colon [24] or bladder carcinoma cell lines [25].

In our study, we found a significant relationship between PIM-1 transcriptional expression and the risk of local recurrence in patients treated with radiotherapy, including patients treated with chemoradiotherapy and bioradiotherapy. Figure 2 of the supplementary material shows the local recurrence-free survival as a function of the PIM-1 expression category depending on the type of treatment used. According to the results of multivariate analysis, the variables that were significantly associated with an increased risk of local recurrence after radiotherapy were hypopharyngeal tumor location, advanced local extension (cT3-4), and high PIM-1 expression.

These differences in local disease control were not ultimately reflected in specific survival, basically due to the possibility of carrying out salvage surgeries after a local recurrence of the tumor. Salvage surgery entails a higher frequency of postoperative complications and morbidity versus similar surgery performed as an initial treatment. The availability of a biomarker with the capacity to assess the sensitivity to radiotherapy would make it possible to personalize the type of treatment according to the tumor's sensitivity profile.

One of the limitations of our study was that it only assessed the transcriptional expression of PIM-1, without providing information about the signaling pathways activated or the existence of post-transcriptional regulation mechanisms. In addition, these results were obtained retrospectively in a single institution from a heterogeneous sample of patients in terms of the location of the primary tumor and the type of treatment used, including patients treated with chemo- and bioradiotherapy. Independent validation of our results would be required before considering PIM-1 transcriptional expression as a predictive biomarker of response to radiotherapy in patients with HNSCC.

\section{Conclusion}

PIM-1 transcriptional expression was significantly related to local disease control in patients with HNSCC treated with radiotherapy. Patients with elevated expression levels of PIM-1 had a significantly higher risk of local recurrence.
Supplementary Information The online version contains supplementary material available at https://doi.org/10.1007/s00405-021-07223-4.

Author contributions All authors contributed substantially to the paper in conception, design, and writing. The publication has been approved by all (co-)authors.

Funding Open Access Funding provided by Universitat Autonoma de Barcelona. This study was supported by a grant from Instituto de Salud Carlos III (FIS PI19/01661 to XL). Fondo Europeo de Desarrollo Regional (FEDER), A Way to Build Europe.

\section{Declarations}

Conflict of interest The authors declare that they do not have any conflict of interest.

Ethical approval The study has been performed in accordance with the ethical standards as laid down in the 1964 Declaration of Helsinki and its later amendments. The study was approved by the Hospital de Sant Pau Ethics Committee for Scientific Research (IIBSP-CCC-14-93).

Informed consent Informed consent was obtained from all individual participants included in the study.

Open Access This article is licensed under a Creative Commons Attribution 4.0 International License, which permits use, sharing, adaptation, distribution and reproduction in any medium or format, as long as you give appropriate credit to the original author(s) and the source, provide a link to the Creative Commons licence, and indicate if changes were made. The images or other third party material in this article are included in the article's Creative Commons licence, unless indicated otherwise in a credit line to the material. If material is not included in the article's Creative Commons licence and your intended use is not permitted by statutory regulation or exceeds the permitted use, you will need to obtain permission directly from the copyright holder. To view a copy of this licence, visit http://creativecommons.org/licenses/by/4.0/.

\section{References}

1. Panchal NK, Sabina EP (2020) A serine/threonine protein PIM kinase as a biomarker of cancer and a target for anti-tumor therapy. Life Sci 255:117866

2. Cheng H, Huang C, Xu X, Hu X, Gong S, Tang G, Song X, Zhang W, Wang J, Chen L, Yang J (2017) PIM-1 mRNA expression is a potential prognostic biomarker in acute myeloid leukemia. $\mathrm{J}$ Transl Med 15:179

3. Szydłowski M, Dębek S, Prochorec-Sobieszek M, Szołkowska M, Tomirotti AM, Juszczyński P, Szumera-Ciećkiewicz A (2021) PIM kinases promote survival and immune escape in primary mediastinal large B-cell lymphoma through modulation of JAKSTAT and NF-KB activity. Am J Pathol 191:567-574

4. Liu HT, Wang N, Wang X, Li SL (2010) Overexpression of PIM-1 is associated with poor prognosis in patients with esophageal squamous cell carcinoma. J Surg Oncol 102:683-688

5. Jiang R, Wang X, Jin Z, Li K (2016) Association of nuclear PIM1 expression with lymph node metastasis and poor prognosis in patients with lung adenocarcinoma and squamous cell carcinoma. J Cancer 7:324-334 
6. Chen J, Tang G (2019) PIM-1 kinase: a potential biomarker of triple-negative breast cancer. Onco Targets Ther 12:6267-6273

7. Xu J, Xiong G, Cao Z, Huang H, Wang T, You L, Zhou L, Zheng L, Hu Y, Zhang T, Zhao Y (2016) PIM-1 contributes to the malignancy of pancreatic cancer and displays diagnostic and prognostic value. J Exp Clin Cancer Res 35:133

8. Peng YH, Li JJ, Xie FW, Chen JF, Yu YH, Ouyang XN, Liang HJ (2013) Expression of PIM-1 in tumors, tumor stroma and tumoradjacent mucosa co-determines the prognosis of colon cancer patients. PLoS ONE 8:e76693

9. Warnecke-Eberz U, Bollschweiler E, Drebber U, Metzger R, Baldus SE, Hölscher AH, Mönig S (2009) Prognostic impact of protein overexpression of the proto-oncogene PIM-1 in gastric cancer. Anticancer Res 29:4451-4455

10. Reiser-Erkan C, Erkan M, Pan Z, Bekasi S, Giese NA, Streit S, Michalski CW, Friess H, Kleeff J (2008) Hypoxia-inducible proto-oncogene PIM-1 is a prognostic marker in pancreatic ductal adenocarcinoma. Cancer Biol Ther 7:1352-1359

11. Kim W, Youn H, Kwon T, Kang J, Kim E, Son B, Yang HJ, Jung Y, Youn B (2013) PIM1 kinase inhibitors induce radiosensitization in non-small cell lung cancer cells. Pharmacol Res 70:90-101

12. Xu D, Allsop SA, Witherspoon SM, Snider JL, Yeh JJ, Fiordalisi JJ, White CD, Williams D, Cox AD, Baines AT (2011) The oncogenic kinase PIM-1 is modulated by K-Ras signaling and mediates transformed growth and radioresistance in human pancreatic ductal adenocarcinoma cells. Carcinogenesis 32:488-495

13. Peltola K, Hollmen M, Maula SM, Rainio E, Ristamäki R, Luukkaa M, Sandholm J, Sundvall M, Elenius K, Koskinen PJ, Grenman R, Jalkanen S (2009) PIM-1 kinase expression predicts radiation response in squamocellular carcinoma of head and neck and is under the control of epidermal growth factor receptor. Neoplasia 11:629-636

14. León X, Orús C, Quer M (2002) Diseño, mantenimiento y explotación de una base de datos oncológica para pacientes con tumores malignos de cabeza y cuello. Acta Otorrinolaringol Esp 53:185-190

15. Chiang WF, Yen CY, Lin CN, Liaw GA, Chiu CT, Hsia YJ, Liu SY (2006) Up-regulation of a serine-threonine kinase proto-oncogene PIM-1 in oral squamous cell carcinoma. Int J Oral Maxillofac Surg 35:740-745

16. Beier UH, Weise JB, Laudien M, Sauerwein H, Görögh T (2007) Overexpression of PIM-1 in head and neck squamous cell carcinomas. Int J Oncol 30:1381-1387
17. Tanaka S, Kitamura T, Higashino F, Hida K, Ohiro Y, Ono M, Kobayashi M, Totsuka Y, Shindoh M (2009) PIM-1 activation of cell motility induces the malignant phenotype of tongue carcinoma. Mol Med Rep 2:313-318

18. Broutian TR, Jiang B, Li J, Akagi K, Gui S, Zhou Z, Xiao W, Symer DE, Gillison ML (2020) Human papillomavirus insertions identify the PIM family of serine/threonine kinases as targetable driver genes in head and neck squamous cell carcinoma. Cancer Lett 476:23-33

19. Karatas OF (2018) Antiproliferative potential of miR-33a in laryngeal cancer Hep-2 cells via targeting PIM1. Head Neck 40:2455-2461

20. Santio NM, Vahakoski RL, Rainio EM, Sandholm JA, Virtanen SS, Prudhomme M, Anizon F, Moreau P, Koskinen PJ (2010) PIM-selective inhibitor DHPCC-9 reveals PIM kinases as potent stimulators of cancer cell migration and invasion. Mol Cancer 9:279

21. Jie W, He QY, Luo BT, Zheng SJ, Kong YQ, Jiang HG, Li RJ, Guo JL, Shen ZH (2012) Inhibition of PIM-1 attenuates the proliferation and migration in nasopharyngeal carcinoma cells. Asian Pac J Trop Med 5:645-650

22. Gu H, Liu M, Ding C, Wang X, Wang R, Wu X, Fan R (2016) Hypoxia-responsive miR-124 and miR-144 reduce hypoxiainduced autophagy and enhance radiosensitivity of prostate cancer cells via suppressing PIM1. Cancer Med 5:1174-1182

23. Zhang X, Sun Y, Wang P, Yang C, Li S (2019) Reduced PIM-1 expression increases chemotherapeutic drug sensitivity in human androgen-independent prostate cancer cells by inducing apoptosis. Exp Ther Med 18:2731-2738

24. Weirauch U, Beckmann N, Thomas M, Grünweller A, Huber K, Bracher F, Hartmann RK, Aigner A (2013) Functional role and therapeutic potential of the PIM-1 kinase in colon carcinoma. Neoplasia 15:783-794

25. Guo S, Mao X, Chen J, Huang B, Jin C, Xu Z, Qiu S (2010) Overexpression of PIM-1 in bladder cancer. J Exp Clin Cancer Res 29:161

Publisher's Note Springer Nature remains neutral with regard to jurisdictional claims in published maps and institutional affiliations. 